\title{
Weak Projective Synchronization in Drive-Response Dynamical Networks with Time-Varying Delay and Parameter Mismatch
}

\author{
Jiang $\mathrm{Xu}^{1}$ and Song Zheng ${ }^{2}$ \\ ${ }^{1}$ School of Mathematics and Physics, Jiangsu University of Science and Technology, Zhenjiang, Jiangsu 212003, China \\ ${ }^{2}$ School of Mathematics and Statistics, Zhejiang University of Finance \& Economics, Hangzhou, Zhejiang 310018, China \\ Correspondence should be addressed to Jiang Xu; jxu078@163.com
}

Received 24 October 2013; Accepted 25 January 2014; Published 15 April 2014

Academic Editor: Jinde Cao

Copyright (C) $2014 \mathrm{~J}$. Xu and S. Zheng. This is an open access article distributed under the Creative Commons Attribution License, which permits unrestricted use, distribution, and reproduction in any medium, provided the original work is properly cited.

\begin{abstract}
This paper investigates the problem of projective synchronization in drive-response dynamical networks (DRDNs) with timevarying delay and parameter mismatch via impulsive control. Owing to projective factor and parameter mismatch, complete projective synchronization cannot be achieved. Therefore, a weak projective synchronization scheme is proposed to ensure that the DRDNs are in a state of synchronization with an error level. Based on the stability analysis of the impulsive functional differential equations, a general method of the weak projective synchronization with the error level is derived in DRDNs. Numerical simulations are provided to verify the correctness and effectiveness of the proposed method and results.
\end{abstract}

\section{Introduction}

A complex dynamical network is a set of coupled nodes interconnected by edges, in which each node represents a dynamical system. The structure of many real systems in nature can be described by the complex dynamical networks, such as social relationship networks, metabolic networks, food chain, Internet, the World Wide Web, power grids, and so on $[1,2]$. This has led to much interest in the studies of the complex dynamical networks. In particular, the synchronization of complex networks has received much attention, and many interesting results on synchronization were derived for various complex networks such as time invariant, timevarying, discrete, and impulsive network models [3-11].

More recently, projective synchronization on dynamical networks has been reported by Hu et al. in [12], in which the projective synchronization with the desired scaling factor can be realized in drive-response dynamical networks. Projective synchronization has become a hot topic and attracted much attention from authors in many fields, including chaotic systems [13-16] and complex dynamical networks [17-20]. In these papers, the authors just consider the projective synchronization in DRDNs with coupled partially linear chaotic systems. However, there are always some mismatches between drive system and response network systems in the real world. Indeed, almost all complex dynamical networks have different nodes, such as the nodes in network community and the Internet, are in general different. In this case, the DRDNs cannot synchronize completely. Nevertheless, when parameter mismatch is small enough, the synchronization error can converge to a small region containing the origin. In [21], the authors investigated the effect of parameter mismatch on lag synchronization of chaotic systems. In [22], the synchronization of a class of coupled chaotic delayed systems with parameters mismatch and stochastic perturbation was studied. In [23], the weak synchronization criterion of coupled delayed chaotic systems with parameters mismatches was obtained. In [24], the authors studied the synchronization of two coupled identical chaotic systems with parameter mismatch via using periodically intermittent control. In [25], the weak projective synchronization of neural networks with mixed time-varying delays and parameter mismatch was discussed. Unfortunately, there exist few results of a weak projective synchronization method for DRDNs with time-varying delay and parameter mismatch. Therefore, it is worth proposing a weak projective synchronization method in which the problems mentioned above are considered. 
Motivated by the above discussions, in this paper, we introduce a drive-response dynamical network with timevarying delay and parameter mismatch. It is known that complete synchronization is destroyed by parameter mismatch and projective factor. We propose the weak projective synchronization properties of this model via impulsive control. Based on the obtained results, one can control the projective synchronization error in a predetermined level. Results of numerical example show the effectiveness of the proposed approach. The rest of this paper is organized as follows. In Section 2, the DRDNs model with parameter mismatch and some preliminaries are given. In Section 3, some criteria for the weak projective synchronization are derived. Numerical simulations are shown in Section 4. The conclusion is finally given in Section 5.

The notation throughout the paper is quite standard. $R$ and $R^{n}$ denote the real number set and $n$-dimensional Euclidean space, respectively. $\|\cdot\|$ stands for either the Euclidean vector norm or its induced matrix 2-norm. $\lambda_{\text {max }}(A)\left(\lambda_{\text {min }}(A)\right)$ represents the maximum (minimum) eigenvalue of the symmetric matrix $A \cdot \mu(A)=\lambda_{\text {max }}\left(A^{T}+A\right)$. sup denotes the upper bound. $I_{n}$ is the identity matrix with order $n$. Matrices, if not explicitly stated, are assumed to have compatible dimensions. $\otimes$ is the Kronecker product of two matrices. $P C\left([-\tau, 0], R^{n}\right)$ denotes the set of all functions of bounded variation and right-continuous on any compact subinterval of $[-\tau, 0]$.

\section{Model Description and Preliminaries}

2.1. Model Description. In this paper, we consider DRDNs with time-varying coupling delays and parameter mismatch as follows:

$$
\begin{aligned}
\dot{x}^{d}(t)= & A^{d} x^{d}(t)+B^{d} f\left(x^{d}(t)\right)+C^{d} g\left(x^{d}(t-\tau(t))\right), \\
\dot{x}_{i}^{r}(t)= & A_{i}^{r} x^{d}(t)+B_{i}^{r} f\left(x_{i}^{r}(t)\right) \\
& +C_{i}^{r} g\left(x_{i}^{r}(t-\tau(t))\right)+\gamma \sum_{j=1}^{N} c_{i j} \Gamma x_{j}^{r}\left(t-\tau_{1}(t)\right),
\end{aligned}
$$

where the superscripts $d$ and $r$ stand for the drive system and response networks, respectively. In (1), $x^{d}(t)=$ $\left(x_{1}^{d}(t), x_{2}^{d}(t), \ldots, x_{n}^{d}(t)\right)^{T} \in R^{n}$ denotes the state vector of the drive system; $A^{d}, B^{d}$, and $C^{d}$ are constant $n \times n$ matrices. $f$ : $R^{n} \rightarrow R^{n}$ and $g: R^{n} \rightarrow R^{n}$ are continuously differentiable vector functions. In $(2), x_{i}^{r}(t)=\left(x_{i 1}^{r}(t), x_{i 2}^{r}(t), \ldots, x_{i n}^{r}(t)\right)^{T} \in$ $R^{n}, i=1,2, \ldots, N$, denotes the state vector of the $i$ th node; $A_{i}^{r}$, $A_{i}^{r}$, and $A_{i}^{r}$ are constant $n \times n$ matrices. $\tau(t), \tau_{1}(t)$ are the timevarying delays. The constant $\gamma>0$ represents the coupling strength of the network, and $\Gamma \in R^{n \times n}$ is the inner-coupling matrix; $C=\left(c_{i j}\right)_{N \times N} \in R^{N \times N}$ is the coupling matrix, standing for the coupling configuration of the network. If there is connection between node $i$ and node $(i \neq j), c_{i j} \neq 0$; otherwise, $c_{i j}=0$. The row sum of $C$ is zero; that is, $c_{i i}=-\sum_{j=1, j \neq i}^{N} c_{i j}$.

2.2. Preliminaries. In order to demonstrate this paper clearly, we give some necessary definitions, assumptions, and lemmas, which are useful in deriving projective synchronization criteria.

Definition 1. The drive system (1) and response dynamical networks (2) are said to be weak projective synchronized with an error level $\varsigma>0$, if there exists a $T \geq 0$ such that $\left\|x_{i}^{r}(t)-\lambda x_{d}(t)\right\| \leq \varsigma$ for all $t \geq T$, where $\lambda$ is a desired scaling factor.

Assumption 2. For any $z_{1}, z_{2} \in R^{n}$, there exist constants $l_{f}>$ $0, l_{g}>0, i=1,2, \ldots, N$, such that $\left\|f\left(z_{1}\right)-f\left(z_{2}\right)\right\| \leq l_{f} \| z_{1}-$ $z_{2}\|,\| g_{i}\left(z_{1}\right)-g_{i}\left(z_{2}\right)\left\|\leq l_{g}\right\| z_{1}-z_{2} \|$.

Assumption 3. $\tau(t)$ and $\tau_{1}(t)$ are the time-varying delay satisfying $0 \leq \tau(t), \tau_{1}(t) \leq \tau$, where $\tau$ is a positive constant. Clearly, this assumption is certainly ensured if the timevarying delay is a constant.

Remark 4. It should be pointed out that in Assumption 3 we do not require that the time-varying delay is differential function with a bound of its derivative, which means that the time-varying delay satisfying Assumption 3 includes a wide range of functions.

Assumption 5. It is assumed that the trajectory of the drive system (1) is bounded with

$$
\Omega=\left\{x^{d}(t) \mid\left\|x^{d}(t)\right\| \leq \delta\right\}, \quad \forall t \geq-\tau .
$$

Remark 6. Assumption 5 is reasonable due to its chaotic feature.

Lemma 7 (see [26]). Let $P \in R^{n \times n}$ be a symmetric positive definite matrix and $P=Q^{T} Q$. For any $x, y \in R^{n}$ and $A \in R^{n \times n}$ such that
(1) $x^{T} A^{T} P A x \leq\left\|Q A Q^{-1}\right\|^{2} x^{T} P x$
(2) $x^{T}\left(A^{T} P+P A\right) x \leq 2 \mu\left(Q A Q^{-1}\right) x^{T} P x$
(3) $\left|x^{T} P y\right| \leq \sqrt{x^{T} P x} \sqrt{y^{T} P y}$.

Lemma 8 (see [26]). Let $0 \leq \tau(t), \tau_{1}(t), \tau_{2}(t), \ldots, \tau_{m}(t) \leq$ $\tilde{\tau}, \tilde{\tau}=\max \left(\tau, \tau_{1}, \tau_{2}, \ldots, \tau_{m}\right), F\left(t, u, \bar{u}_{1}, \ldots, \bar{u}_{m}\right): R^{+} \times$ $m+1$

$\overbrace{R \times \cdots \times R} \rightarrow R$ be nondecreasing in $\bar{u}_{i}$ for each fixed $(t$, $\left.u, \bar{u}_{1}, \ldots, \bar{u}_{i-1}, \bar{u}_{i+1}, \bar{u}_{m}\right), i=1,2, \ldots, m$, and let $I_{k}(u): R \rightarrow$ $R$ be nondecreasing in $u$. Suppose that $u(t)$ and $v(t)$ satisfy

$$
\begin{gathered}
D^{+} u(t) \leq F\left(t, u(t), u_{1}\left(t-\tau_{1}(t)\right), \ldots, u_{m}\left(t-\tau_{m}(t)\right)\right), \\
t \geq 0, \\
u\left(t_{k}\right) \leq I_{k} u\left(t_{k}\right), \quad k \in N, \\
D^{+} v(t)>F\left(t, v(t), v_{1}\left(t-\tau_{1}(t)\right), \ldots, v_{m}\left(t-\tau_{m}(t)\right)\right), \\
v\left(t_{k}^{+}\right) \geq I_{k} v\left(t_{k}\right), \quad k \in N ;
\end{gathered}
$$


then $u(t) \leq v(t)$ for $-\tau \leq t \leq 0$ implies that $u(t) \leq v(t)$ for $t \geq 0$, where the right and upper Dini's derivative $D^{+} u(t)$ is defined as $D^{+} u(t)=\overline{\lim }_{h \rightarrow 0^{+}}((u(t+h)-u(t)) / h)$.

The aim of this paper is to discuss the weak projective synchronization in the DRDNs with time-varying delay and parameters mismatches. We choose the impulsive controller $B_{i k}$ which is a $n \times n$ constant matrix. Thus, the drive system (1) and response networks (2) can be rewritten as the following impulsive differential equations:

$$
\begin{gathered}
\dot{x}^{d}(t)=A^{d} x^{d}(t)+B^{d} f\left(x^{d}(t)\right)+C^{d} g\left(x^{d}(t-\tau(t))\right), \\
\dot{x}_{i}^{r}(t)=A_{i}^{r} x^{d}(t)+B_{i}^{r} f\left(x_{i}^{r}(t)\right)+C_{i}^{r} g\left(x_{i}^{r}(t-\tau(t))\right) \\
+\gamma \sum_{j=1}^{N} c_{i j} \Gamma x_{j}^{r}\left(t-\tau_{1}(t)\right), \quad t \neq t_{k}, \\
\Delta x_{i}^{r}=x_{i}^{r}\left(t_{k}^{+}\right)-x_{i}^{r}\left(t_{k}^{-}\right)=B_{i k}\left[x_{i}^{r}-\lambda x^{d}\right], \\
t=t_{k}, k=1,2, \ldots,
\end{gathered}
$$

where the impulsive time instants $t_{k}$ satisfy $0=t_{0}<t_{1}<t_{2}<$ $\cdots<t_{k}<\cdots$ and $\lim _{k \rightarrow \infty} t_{k}=+\infty, x_{i}^{r}\left(t_{k}^{+}\right)=\lim _{t \rightarrow t_{k}^{+}} x_{i}^{r}(t)$, $x_{i}^{r}\left(t_{k}^{-}\right)=\lim _{t \rightarrow t_{k}^{-}} x_{i}^{r}(t)$. Moreover, any solution of (5) is left continuous at each $t_{k}$; that is, $x_{i}^{r}\left(t_{k}^{-}\right)=x_{i}^{r}\left(t_{k}\right)$.

Letting $e_{i}(t)=x_{i}^{r}(t)-\lambda x^{d}(t)$, then the synchronization error system between the drive system and the response network can be written as

$$
\begin{gathered}
\dot{e}_{i}(t)=A_{i}^{r} e_{i}(t)+B_{i}^{r} \tilde{f}\left(e_{i}(t)\right)+C_{i}^{r} \tilde{g}\left(e_{i}(t-\tau(t))\right) \\
+c \sum_{j=1}^{N} c_{i j} \Gamma e_{j}\left(t-\tau_{1}(t)\right) \\
+\tilde{h}_{i}\left(x^{d}(t), \tau(t), \lambda\right), \quad t \neq t_{k}, \\
\Delta e_{i}=B_{i k} e_{i}, \quad t=t_{k},
\end{gathered}
$$

where $\tilde{f}\left(e_{i}(t)\right)=f\left(x_{i}^{r}(t)\right)-f\left(\lambda x^{d}(t)\right), \tilde{g}\left(e_{i}(t-\tau(t))\right)=$ $g\left(x_{i}^{r}(t-\tau(t))\right)-g\left(\lambda x^{d}(t-\tau(t))\right)$ and $\widetilde{h}_{i}\left(x^{d}(t), \tau(t), \lambda\right)=\lambda\left(A_{i}^{r}-\right.$ A) $x^{d}(t)+B_{i}^{r} f\left(\lambda x^{d}(t)\right)-\lambda B^{d} f\left(x^{d}(t)\right)+C_{i}^{r} g\left(\lambda x^{d}(t-\tau(t))\right)-$ $\lambda C^{d} g\left(x^{d}(t-\tau(t))\right)$.

Let $e(t)=\left(e_{1}^{T}(t), e_{2}^{T}(t), \ldots, e_{N}^{T}(t)\right)^{T}$, rewriting (6) in its compact form

$$
\begin{aligned}
\dot{e}(t)= & \left(I_{N} \otimes A_{i}^{r}\right) e(t)+\left(I_{N} \otimes B_{i}^{r}\right) F(e(t))+\left(I_{N} \otimes C_{i}^{r}\right) \\
& \times G(e(t-\tau(t)))+\gamma(C \otimes \Gamma) e\left(t-\tau_{1}(t)\right) \\
& +H\left(x^{d}(t), \tau(t), \lambda\right), \quad t \neq t_{k}, \\
e\left(t_{k}^{+}\right)= & \left(I_{N n}+\left(I_{N} \otimes B_{i k}\right)\right) e\left(t_{k}\right), \quad t=t_{k}, k=1,2, \ldots,
\end{aligned}
$$

where $F(e(t))=\left(\tilde{f}^{T}\left(e_{1}(t)\right), \tilde{f}^{T}\left(e_{2}(t)\right), \ldots, \tilde{f}^{T}\left(e_{N}(t)\right)\right)^{T}$, $G(e(t-\tau(t)))=\left(\tilde{g}^{T}\left(e_{1}(t-\tau(t))\right), \tilde{g}^{T}\left(e_{2}(t-\tau(t))\right), \ldots\right.$,
$\left.\tilde{g}^{T}\left(e_{N}(t-\tau(t))\right)\right)^{T}$ and $H\left(x^{d}(t), \tau(t), \lambda\right)=\left(\tilde{h}_{1}^{T}\left(x^{d}(t)\right.\right.$, $\left.\tau(t), \lambda), \ldots, \widetilde{h}_{N}^{T}\left(x^{d}(t), \tau(t), \lambda\right)\right)^{T}$.

The initial condition of the error system (7) is defined as $e(s)=\phi(s),-\tau \leq s<0$, where $\phi(\cdot) \in C\left([-\tau, 0], R^{n}\right) .\|\phi\|=$ $\sup _{-\tau<s<0}\|\phi(s)\|$ is used to denote the norm of a function $\phi \in C\left([-\tau, 0], R^{n}\right)$. It is assumed that (7) has a unique solution with respect to initial condition.

\section{Main Results}

In this section, by combining the stability analysis of impulsive functional differential equations, some sufficient conditions for weak projective synchronization in drive-response dynamical networks with time-varying delay and parameter mismatch under impulsive control are given below.

Theorem 9. Under Assumptions 2, 3, and 5, let a nonsingular matrix $Q \in R^{N n \times N n}, 0<\rho=\sup \left\{t_{k}-t_{k-1}\right\}<\infty$, and $\sup _{t \geq 0}\left\|H\left(t, x^{d}(t), \lambda\right)\right\| \leq \omega<\infty$. If the following inequalities hold

$$
\begin{gathered}
\left\|I_{N n}+Q\left(I_{N} \otimes B_{i k}\right) Q^{-1}\right\| \leq \beta, \quad 0<\beta<1, \\
\frac{2 \ln \beta}{\rho}+a+\beta^{-2} b+\beta^{-2} c<0,
\end{gathered}
$$

where $\alpha=-a-(2 \ln \beta / \rho), a=\max _{1<i<N}\left[\left(\mu\left[Q\left(I_{N} \otimes A_{i}^{r}\right) Q^{-1}\right]\right)+\right.$ $\left(2 l_{f} \lambda_{\max }(P)\left\|I_{N} \otimes B_{i}^{r}\right\|+l_{g} \lambda_{\max }(P)\left\|I_{N} \otimes C_{i}^{r}\right\| \zeta\right) / \lambda_{\min }(P)+$ $\left.\|P\|^{2} / \xi \lambda_{\min }(P)+\gamma\right], b=l_{g} \lambda_{\max }(P)\left\|I_{N} \otimes C_{i}^{r}\right\| / \zeta \lambda_{\min }(P), c=$ $\gamma\left\|Q(C \otimes \Gamma) Q^{-1}\right\|^{2}, \zeta$ and $\xi$ are positive constants. $\nu>0$ is a unique solution of $\nu-\alpha+\beta^{-2} b e^{\nu \tau}+\beta^{-2} c e^{\nu \tau_{1}}=0$. Then, the error system (7) can converge globally exponentially to the small region $M$ containing the origin, where $M=\left\{e(t) \in R^{N n}\right.$ | $\left.\|e(t)\| \leq(\omega / \beta) \sqrt{\xi /\left(\alpha-\beta^{-2} b-\beta^{-2} c\right)}\right\}$, which implies the weak projective synchronization in DRDNs is achieved.

Proof. Consider the following Lyapunov functional:

$$
V(t)=e^{T}(t) P e(t)
$$

where $P$ is a symmetric matrix and $P=Q^{T} Q$.

For $t \neq t_{k}$, the time derivative of $V(t)$ along the trajectories of (7) is

$$
\begin{aligned}
\dot{V}(t)=2 e^{T}(t) P \dot{e}( & \\
=2 e^{T}(t) P[ & \left(I_{N} \otimes A_{i}^{r}\right) e(t)+\left(I_{N} \otimes B_{i}^{r}\right) F(e(t)) \\
& +\left(I_{N} \otimes C_{i}^{r}\right) G(e(t-\tau(t))) \\
& +\gamma(C \otimes \Gamma) e\left(t-\tau_{1}(t)\right) \\
& \left.+H\left(x^{d}(t), \tau(t), \lambda\right)\right]
\end{aligned}
$$




$$
\begin{aligned}
= & e^{T}(t)\left[\left(I_{N} \otimes A_{i}^{r}\right)^{T} P+P\left(I_{N} \otimes A_{i}^{r}\right)\right] e(t) \\
& +2 \gamma e^{T}(t) P(C \otimes \Gamma) e\left(t-\tau_{1}(t)\right) \\
& +2 e^{T}(t) P\left(I_{N} \otimes B_{i}^{r}\right) F(e(t)) \\
& +2 e^{T}(t) P\left(I_{N} \otimes C_{i}^{r}\right) G(e(t-\tau(t))) \\
& +2 e^{T}(t) P H\left(x^{d}(t), \tau(t), \lambda\right) .
\end{aligned}
$$

From Lemmas 7-8 and Assumption 2, it is clear that

$\dot{V}(t)$

$$
\begin{aligned}
\leq & \mu\left(Q\left(I_{N} \otimes A_{i}\right) Q^{-1}\right) e^{T}(t) P e(t)+\frac{\|P\|^{2}}{\xi}\|e(t)\|^{2} \\
& +\xi\left\|H\left(x^{d}(t), \tau(t), \lambda\right)\right\|^{2} \\
& +2 l_{f} \lambda_{\max }(P)\left\|I_{N} \otimes B_{i}^{r}\right\|\|e(t)\|^{2} \\
& +2 l_{g} \lambda_{\max }(P)\left\|I_{N} \otimes C_{i}^{r}\right\|\|e(t)\|\|e(t-\tau(t))\| \\
& +2 \gamma \sqrt{e^{T}(t) P e(t)} \\
& \times \sqrt{e^{T}\left(t-\tau_{1}(t)\right)(C \otimes \Gamma)^{T} P(C \otimes \Gamma) e\left(t-\tau_{1}(t)\right)} \\
\leq & \max _{1 \leq i \leq N}\left[\left(\mu\left[Q\left(I_{N} \otimes A_{i}^{r}\right) Q^{-1}\right]\right)\right.
\end{aligned}
$$$$
+\frac{2 l_{f} \lambda_{\max }(P)\left\|I_{N} \otimes B_{i}^{r}\right\|+l_{g} \lambda_{\max }(P)\left\|I_{N} \otimes C_{i}^{r}\right\| \zeta}{\lambda_{\min }(P)}
$$$$
\left.+\frac{\|P\|^{2}}{\xi \lambda_{\min }(P)}+\gamma\right] e^{T}(t) P e(t)
$$$$
+\frac{l_{g} \lambda_{\max }(P)\left\|I_{N} \otimes C_{i}^{r}\right\|}{\zeta \lambda_{\min }(P)} e^{T}(t-\tau(t)) P e(t-\tau(t))
$$$$
+\gamma\left\|Q(C \otimes \Gamma) Q^{-1}\right\|^{2} e^{T}\left(t-\tau_{1}(t)\right)
$$$$
\times P e\left(t-\tau_{1}(t)\right)+\xi \omega^{2}
$$$$
=a V(t)+b V(t-\tau(t))+c V\left(t-\tau_{1}(t)\right)+\xi \omega^{2} .
$$

When $t=t_{k}$, one gets

$$
\begin{aligned}
V\left(t_{k}^{+}\right)= & e^{T}\left(t_{k}\right)\left(I_{N n}+\left(I_{N} \otimes B_{i k}\right)\right)^{T} \\
& \times P\left(I_{N n}+\left(I_{N} \otimes B_{i k}\right)\right) e\left(t_{k}\right) \\
\leq & \left\|I_{N n}+Q\left(I_{N} \otimes B_{i k}\right) Q^{-1}\right\|^{2} e^{T}\left(t_{k}\right) P e\left(t_{k}\right) \\
= & \beta^{2} V\left(t_{k}\right), \quad k=1,2, \ldots
\end{aligned}
$$

For any $\varepsilon>0$, let $v(t)$ be a unique solution of the following impulsive delayed system:

$$
\begin{gathered}
\dot{v}(t)=a v(t)+b v(t-\tau(t))+c v\left(t-\tau_{1}(t)\right)+\xi \omega^{2}+\varepsilon, \\
t \neq t_{k}, \\
v\left(t_{k}^{+}\right)=\beta^{2} v\left(t_{k}\right), \quad k \in N, \\
v(s)=\lambda_{\max }(P)\|\phi(s)\|^{2}, \quad-\tau \leq s \leq 0 .
\end{gathered}
$$

From Lemma 8 and $V(t) \leq\|\phi(t)\|^{2}$ for $-\tau \leq t \leq 0$, we conclude that $V(t) \leq v(t)$, for $t>0$.

The trivial solution of the comparison system is

$$
\begin{gathered}
v(t)=w(t, 0) v(0) \\
+\int_{0}^{t} w(t, s)\left(b v(s-\tau(s))+c v\left(s-\tau_{1}(s)\right)\right. \\
\left.+\xi \omega^{2}+\varepsilon\right) d s
\end{gathered}
$$

where $w(t, s), 0 \leq s \leq t$ is Cauchy matrix of the linear impulsive system.

Since $0<\beta<1, t_{k}-t_{k-1} \leq \rho$, one has

$$
\begin{aligned}
w(t, s) & =e^{a(t-s)} \prod_{s<t_{k} \leq t} \beta^{2} \\
& \leq e^{(-\alpha-2 \ln \beta / \rho)(t-s)} \beta^{2(((t-s) / \rho)-1)} \\
& \leq \beta^{-2} e^{-\alpha(t-s)}, \quad 0 \leq s \leq t .
\end{aligned}
$$

Let $\sigma=\beta^{-2} \lambda_{\max }(P) \sup _{-\tau \leq s \leq 0}\|\phi(s)\|^{2}$, from (15) and (16), one has

$$
\begin{aligned}
v(t) \leq & \beta^{-2} e^{-\alpha t} v(0) \\
& +\int_{0}^{t} e^{-\alpha(t-s)} \beta^{-2} \\
& \quad \times\left[b v(s-\tau(s))+c v\left(s-\tau_{1}(s)\right)+\xi \omega^{2}+\varepsilon\right] d s \\
\leq & \sigma e^{-\alpha t} \\
& +\int_{0}^{t} e^{-\alpha(t-s)} \beta^{-2} \\
& \quad \times\left[b v(s-\tau(s))+c v\left(s-\tau_{1}(s)\right)+\xi \omega^{2}+\varepsilon\right] d s .
\end{aligned}
$$

Denote $\varphi(\nu)=\nu-\alpha+\beta^{-2} b e^{\nu \tau}+\beta^{-2} c e^{\nu \tau_{1}}$; from (9), one has $\alpha>0, b>0, c>0, \alpha-\beta^{-2} b-\beta^{-2} c>0, \varphi(0)<0$, $\varphi(+\infty)>0, \varphi^{\prime}(\nu)=1+\beta^{-2} b \tau e^{\nu \tau}+\beta^{-2} c \tau_{1} e^{\nu \tau_{1}}>0$; then $\varphi(\nu)=0$ has a unique solution $\nu>0$. Since $\beta^{-2}\left(\xi \omega^{2}+\varepsilon\right)>0$, $\nu>0, \alpha-\beta^{-2} b-\beta^{-2} c>0$, and $\beta^{-2}>1$; we derive that

$$
v(t) \leq \beta^{-2} \sup _{-\tau \leq s \leq 0} v(s)<\sigma e^{-v t}+\frac{\xi \omega^{2}+\varepsilon}{\beta^{2} \alpha-b-c}, \quad-\tau \leq t \leq 0 .
$$


In the following, we will prove that the following inequality holds:

$$
v(t)<\sigma e^{-v t}+\frac{\xi \omega^{2}+\varepsilon}{\beta^{2} \alpha-b-c}, \quad t>0 .
$$

If it is not true, there exists a $t^{*}>0$ such that

$$
\begin{gathered}
v\left(t^{*}\right) \geq \sigma e^{-v t^{*}}+\frac{\xi \omega^{2}+\varepsilon}{\beta^{2} \alpha-b-c}, \\
v(t)<\sigma e^{-v t}+\frac{\xi \omega^{2}+\varepsilon}{\beta^{2} \alpha-b-c}, \quad t<t^{*} .
\end{gathered}
$$

From Assumption 3, (17), and (21), we obtain

$$
\begin{aligned}
& v\left(t^{*}\right) \\
& \leq \sigma e^{-\alpha t^{*}}+\int_{0}^{t^{*}} e^{-\alpha\left(t^{*}-s\right)} \beta^{-2} \\
& \times\left[b v(s-\tau(s))+c v\left(s-\tau_{1}(s)\right)+\xi \omega^{2}+\varepsilon\right] d s \\
& <e^{-\alpha t^{*}}\left(\sigma+\frac{\xi N \omega^{2}+\varepsilon}{\beta^{2} \alpha-b-c}\right) \\
& +e^{-\alpha t^{*}} \int_{0}^{t^{*}} e^{\alpha s} \beta^{-2} b\left(\sigma e^{-\nu(s-\tau(s))}+\frac{\xi \omega^{2}+\varepsilon}{\beta^{2} \alpha-b-c}\right) d s \\
& +e^{-\alpha t^{*}} \int_{0}^{t^{*}} e^{\alpha s} \beta^{-2} c\left(\sigma e^{-v\left(s-\tau_{1}(s)\right)}+\frac{\xi \omega^{2}+\varepsilon}{\beta^{2} \alpha-b-c}\right) d s \\
& +e^{-\alpha t^{*}} \int_{0}^{t^{*}} e^{\alpha s} \beta^{-2}\left(\xi \omega^{2}+\varepsilon\right) d s \\
& \leq e^{-\alpha t^{*}}\left\{\sigma+\frac{\xi \omega^{2}+\varepsilon}{\beta^{2} \alpha-b-c}+\beta^{-2} \sigma\left(b e^{\nu \tau}+c e^{\nu \tau_{1}}\right) e^{\nu \tau}\right. \\
& \times \int_{0}^{t^{*}} e^{(a-v) s} d s \\
& +\left(\frac{\beta^{-2}(b+c)\left(\xi \omega^{2}+\varepsilon\right)}{\beta^{2} \alpha-b-c}+\frac{\xi \omega^{2}+\varepsilon}{\beta^{2}}\right) \\
& \left.\times \int_{0}^{t^{*}} e^{a s} d s\right\} \\
& =\sigma e^{-\lambda t^{*}}+\frac{\xi \omega^{2}+\varepsilon}{\beta^{2} \alpha-b-c}
\end{aligned}
$$

which contradicts with (20). Consequently, (19) holds.

Let $\varepsilon \rightarrow 0$; then one obtains

$$
V(t)=\|e(t)\|^{2} \leq v(t) \leq \sigma e^{-\lambda t}+\frac{\xi \omega^{2}}{\beta^{2} \alpha-b-c} .
$$

Thus, one has

$$
\|e(t)\| \leq \sqrt{\sigma} e^{-(v / 2) t}+\frac{\omega}{\beta} \sqrt{\frac{\xi}{\alpha-\beta^{-2} b-\beta^{-2} c}} .
$$

When $t \rightarrow \infty$, the synchronization error system (7) converges exponentially to a small region $M$ containing the ori$\operatorname{gin}: M=\left\{e(t) \in R^{N n} \mid\|e(t)\| \leq(\omega / \beta) \sqrt{\xi /\left(\alpha-\beta^{-2} b-\beta^{-2} c\right)}\right\}$, which implies that the DRDNs achieve the weak projective synchronization. The proof is completed.

By further estimating the value of $H\left(x^{d}(t), \tau(t), \lambda\right)$ and selecting $\xi$ appropriately, we have Corollary 10.

Corollary 10. Under Assumptions 2, 3, and 5, suppose a nonsingular matrix $Q \in R^{N n \times N n}, 0<\rho=\sup \left\{t_{k}-t_{k-1}\right\}<\infty$, and $\delta|\lambda|\left[\left\|A_{i}^{r}-A\right\|+\left(\left\|B_{i}^{r}\right\|+\left\|B^{d}\right\|\right) l_{f}+\left(\left\|C_{i}^{r}\right\|+\left\|C^{d}\right\|\right) l_{g}\right] \leq$ $\omega_{i}$. For given synchronization scaling factor $\lambda$, if the following inequalities hold

$$
\begin{gathered}
\left\|I_{N n}+Q\left(I_{N} \otimes B_{i k}\right) Q^{-1}\right\| \leq \beta, \quad 0<\beta<1, \\
\frac{2 \ln \beta}{\rho}+a+\beta^{-2} b+\beta^{-2} c<0,
\end{gathered}
$$

where $\alpha=-a-(2 \ln \beta / \rho), b=l_{g} \lambda_{\max }(P)\left\|I_{N} \otimes C_{i}^{r}\right\| / \zeta \lambda_{\min }(P)$, $a=\max _{1 \leq i \leq N}\left[\left(\mu\left[Q\left(I_{N} \otimes A_{i}^{r}\right) Q^{-1}\right]\right)+\gamma+\left(\|P\|^{2} / \xi \lambda_{\min }(P)\right)+\right.$ $\left.\left(\left(2 l_{f} \lambda_{\max }(P)\left\|I_{N} \otimes B_{i}^{r}\right\|+l_{g} \lambda_{\text {max }}(P)\left\|I_{N} \otimes C_{i}^{r}\right\| \zeta\right) / \lambda_{\text {min }}(P)\right)\right]$, $c=\gamma\left\|Q(C \otimes \Gamma) Q^{-1}\right\|^{2}$ and $\nu>0$ is an unique solution of $v-\alpha+\beta^{-2} b e^{\nu \tau}+\beta^{-2} c e^{\nu \tau_{1}}=0$, then, the error system (7) can converge globally exponentially to the small region $M$ containing the origin, where $M=\left\{e(t) \in R^{N n} \mid\|e(t)\| \leq\right.$ $\left.(\omega / \beta) \sqrt{\left(\xi /\left(\alpha-\beta^{-2} b-\beta^{-2} c\right)\right)}\right\}$, which implies that the weak projective synchronization in DRDNs is achieved.

Remark 11. For simplicity, we consider the equidistant impulsive interval $t_{k}-t_{k-1}=\Delta$, and the impulsive control gain matrix $B_{k}=b_{0} I_{n}, k=1,2, \ldots$, in Theorem 9. If the following condition holds $\Delta<-2\left(1+b_{0}\right)^{2} \ln \left|1+b_{0}\right| /\left(\left(1+b_{0}\right)^{2} a+b+c\right)$, $-2<b_{0}<0$, then the DRDNs achieve weak projective synchronization.

\section{Numerical Simulation}

In this section, an example is presented to show the effectiveness of the proposed scheme. To show the advantage of the criteria based on matrix measure, a scalar Ikeda oscillator is investigated in the context of weak projective synchronization in the following example.

The dynamics of Ikeda oscillator is described by

$$
\dot{x}=-d x+e \sin (x(t-\tau(t))) \text {. }
$$

System (26) exhibits chaotic behavior when $d=1, e=4$ and $\tau(t)=2$, as shown in Figure 1. It is known that the chaotic attractor of system (27) is contained in the set $\Omega=\{x \in R \mid$ $|x| \leq 4\}$. 


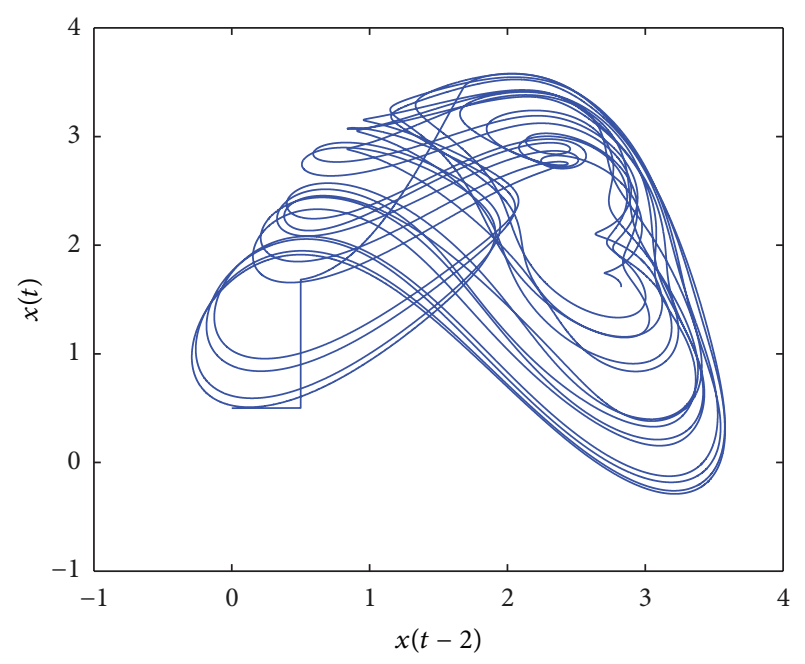

FIGURE 1: Chaotic attractor of system (27).

The corresponding response network systems with parameter mismatch are given by

$$
\begin{aligned}
\dot{x}_{i}^{r}(t)= & -(d-0.001 i) x_{i}^{r}(t)+(e-0.001 i) \sin \left(x_{i}^{r}(t-\tau(t))\right) \\
& +\gamma \sum_{j=1}^{4} c_{i j} \Gamma x_{j}^{r}\left(t-\tau_{1}(t)\right) .
\end{aligned}
$$

Then, the controlled DRDNs are described as follows:

$$
\begin{gathered}
\dot{x}^{d}=-x^{d}+4 \sin \left(x^{d}(t-\tau(t))\right), \\
\dot{x}_{i}^{r}(t)=-(1-0.001 i) x_{i}^{r}(t)+(4-0.001 i) \sin \left(x_{i}^{r}(t-\tau(t))\right) \\
+\gamma \sum_{j=1}^{4} c_{i j} \Gamma x_{j}^{r}\left(t-\tau_{1}(t)\right) \quad t \neq t_{k}, i=1,2,3,4, \\
\Delta e_{i}=b_{0} e_{i}\left(t_{k}^{-}\right), \quad t=t_{k}, \quad k=1,2, \ldots,
\end{gathered}
$$

choosing the coupling configuration matrix

$$
C=\left(\begin{array}{cccc}
-2 & 1 & 1 & 0 \\
1 & -1 & 0 & 0 \\
2 & 1 & -3 & 0 \\
0 & 0 & 1 & -1
\end{array}\right) .
$$

In the numerical simulations, we assume that $\xi=1$, $\zeta=\gamma=0.1, b_{0}=-0.9,\left(1+b_{0}\right)^{2}=0.01>0 . \Gamma=$ $P=1, Q=I_{N n}$. The two coupling delays are $\tau(t)=2$ and $\tau_{1}(t)=2+0.02 \sin t$, respectively. After calculations, getting $a=38.092, b=0.3999, c=1.814$, one has $\Delta<$ 0.0177 . Taking the impulsive interval $\Delta=t_{k+1}-t_{k}=0.01$, then, it is easy to verify that all conditions in Corollary 10 are satisfied. The projective synchronization error is defined by $\|e(t)\|=\sqrt{\left(x_{i 1}-\lambda x_{1}\right)^{2}+\left(y_{i 2}-\lambda y_{2}\right)^{2}}, i=1,2,3,4$. When $\lambda=$ -0.5 , as shown in Figures 2-4. Figure 2 shows attractors of

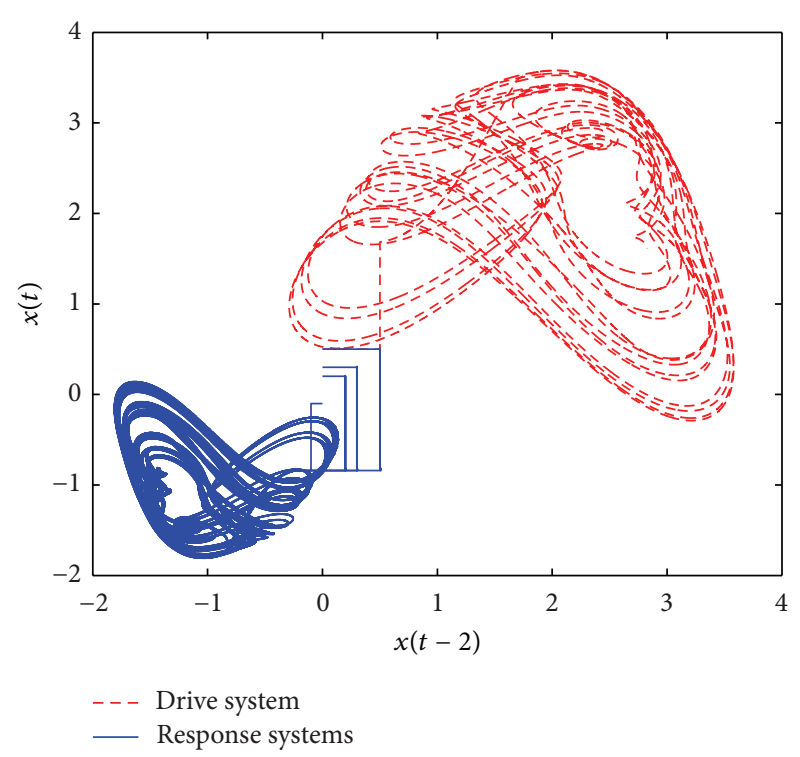

FIGURE 2: Chaotic attractors of systems (27) and (28) with $\lambda=-0.5$.

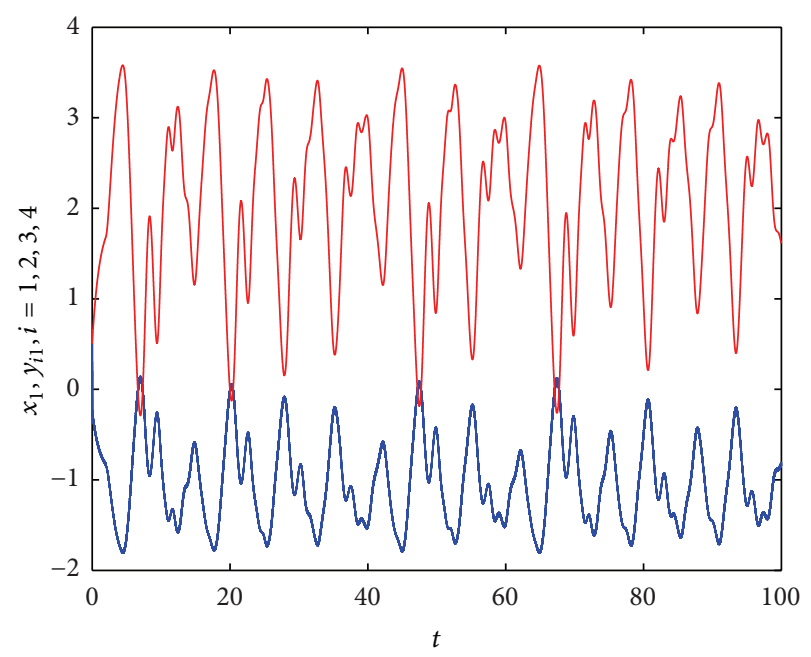

FIGURE 3: The trajectories of state variables with $\lambda=-0.5$.

the DRDNs network model. Figure 3 displays time evolutions of state trajectories of the controlled DRDNs (29). The evolution process of the error does not converge to zero as shown in Figure 4; from Figure 4, it is easy to see that the projective synchronization is not achieved. The numerical results show that the impulsive controlling scheme for the drive-response coupled dynamical network model with timevarying delays is effective.

\section{Conclusion}

In this paper, the problem of weak projective synchronization in DRDNs with time-varying coupling delay and parameter mismatch has been investigated by employing impulsive control scheme. Some criteria for realizing the weak projective synchronization are established based on the stability analysis 


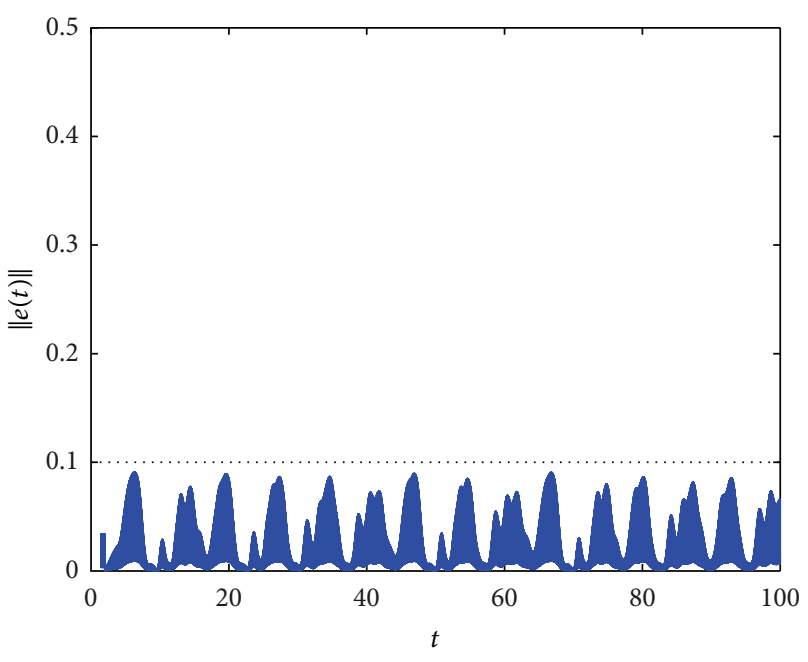

Figure 4: Time evolution of $\|e(t)\|$ of coupled networks (29).

of impulsive functional differential equations. Moreover, the DRDNs can be synchronized exponentially within a small error; the error upper bound of weak projective synchronization is estimated easily by the theoretical criteria. Finally, the numerical examples show the effectiveness of the proposed results. However, the results of theoretical analysis in this paper are still conservative. Meanwhile, since the surrounding environment is complex variable, it is desirable to investigate weak projective synchronization problem for complex dynamical networks with noise, stochastic disturbances, and so on, so we will further investigate these problems in the future.

\section{Conflict of Interests}

The authors declare that they have no conflict of interests.

\section{Authors' Contributions}

Jiang $\mathrm{Xu}$ carried out the main part of this paper. Song Zheng participated in the discussion and corrected the main theorem. All authors read and approved the final paper.

\section{Acknowledgments}

This work was jointly supported by the National Science Foundation of China (Grants nos. 11102076 and 11202085), the Society Science Foundation from Ministry of Education of China (Grant no. 12YJAZH002), the Natural Science Foundation of Zhejiang Province (Grant no. LY13F030016), and the Foundation of Zhejiang Provincial Education Department (Grant no. Y201328316).

\section{References}

[1] D. J. Watts and S. H. Strogatz, "Collective dynamics of "smallworld” networks," Nature, vol. 393, no. 6684, pp. 440-442, 1998.
[2] A.-L. Barabási and R. Albert, "Emergence of scaling in random networks," Science, vol. 286, no. 5439, pp. 509-512, 1999.

[3] M. Chavez, D.-U. Hwang, A. Amann, H. G. E. Hentschel, and S. Boccaletti, "Synchronization is enhanced in weighted complex networks," Physical Review Letters, vol. 94, no. 21, Article ID 218701, 4 pages, 2005.

[4] J. Cao, Z. Wang, and Y. Sun, "Synchronization in an array of linearly stochastically coupled networks with time delays," Physica A, vol. 385, no. 2, pp. 718-728, 2007.

[5] P. de Lellis, M. di Bernardo, and F. Garofalo, "Synchronization of complex networks through local adaptive coupling," Chaos, vol. 18, no. 3, Article ID 037110, 2008.

[6] J. Cao and L. Li, "Cluster synchronization in an array of hybrid coupled neural networks with delay," Neural Networks, vol. 22, no. 4, pp. 335-342, 2009.

[7] Q. Zhu and J. Cao, "Adaptive synchronization of chaotic CohenCrossberg neural networks with mixed time delays," Nonlinear Dynamics, vol. 61, no. 3, pp. 517-534, 2010.

[8] W. Sun, J. Zhang, and C. Li, "Synchronization analysis of two coupled complex networks with time delays," Discrete Dynamics in Nature and Society, vol. 2011, Article ID 209321, 12 pages, 2011.

[9] W. Sun, Y. Yang, C. Li, and Z. Liu, "Synchronization inside complex dynamical networks with double time-delays and nonlinear inner-coupling functions," International Journal of Modern Physics B, vol. 25, no. 11, pp. 1531-1541, 2011.

[10] J. Cao, A. Alofi, A. Al-Mazrooei, and A. Elaiw, "Synchronization of switched interval networks and applications to chaotic neural networks," Abstract and Applied Analysis, vol. 2013, Article ID 940573, 11 pages, 2013.

[11] W. Sun, "Random walks on generalized Koch networks," Physica Scripta, vol. 88, no. 4, Article ID 045006, 2013.

[12] M. Hu, Y. Yang, Z. Xu, R. Zhang, and L. Guo, "Projective synchronization in drive-response dynamical networks," Physica A, vol. 381, no. 1-2, pp. 457-466, 2007.

[13] R. Mainieri and J. Rehacek, "Projective synchronization in three-dimensional chaotic systems," Physical Review Letters, vol. 82, no. 15, pp. 3042-3045, 1999.

[14] Z.-L. Wang, "Projective synchronization of hyperchaotic Lü system and Liu system," Nonlinear Dynamics, vol. 59, no. 3, pp. 455-462, 2010.

[15] J. Cao, D. W. C. Ho, and Y. Yang, "Projective synchronization of a class of delayed chaotic systems via impulsive control," Physics Letters A, vol. 373, no. 35, pp. 3128-3133, 2009.

[16] M. Stivastava, S. Agrawal, and S. Das, "Adaptive projective synchronization between different chaotic systems with parametric uncertainties and external disturbances," Pramana, vol. 81, no. 3, pp. 417-437, 2013.

[17] Y. Zhao and Y. Yang, "The impulsive control synchronization of the drive-response complex system," Physics Letters A, vol. 372, no. 48, pp. 7165-7171, 2008.

[18] X. Xu, Y. Gao, Y. Zhao, and Y. Yang, "The impulsive control of the projective synchronization in the drive-response dynamical networks with coupling delay," in Advances in Neural Networks, vol. 6063 of Lecture Notes in Computer Science, pp. 520-527, Springer, Berlin, Gremany, 2010.

[19] S. Zheng, "Adaptive-impulsive control of the projective synchronization in drive-response complex dynamical networks with time-varying coupling," Mathematical Problems in Engineering, vol. 2012, Article ID 501843, 12 pages, 2012.

[20] S. Zheng, "Analyzing projective synchronization on different scaling factors in a drive-response coupling dynamical network 
with time-varying delays," Nonlinear Dynamics, vol. 70, no. 1, pp. 709-719, 2012.

[21] L. Wang, Z. Yuan, X. Chen, and Z. Zhou, "Lag synchronization of chaotic systems with parameter mismatches," Communications in Nonlinear Science and Numerical Simulation, vol. 16, no. 2, pp. 987-992, 2011.

[22] C. Liu, C. D. Li, and C. J. Li, "Quasi-synchronization of delayed chaotic systems with parameters mismatch and stochastic perturbation," Communications in Nonlinear Science and Numerical Simulation, vol. 16, no. 10, pp. 4108-4119, 2011.

[23] W. He, F. Qian, Q. Han, and J. Cao, "Lag quasi-synchronization of coupled delayed systems with parameter mismatch," IEEE Transactions on Circuits and Systems, vol. 58, no. 6, pp. 13451357, 2011.

[24] W. Zhang, J. Huang, and P. Wei, "Weak synchronization of chaotic neural networks with parameter mismatch via periodically intermittent control," Applied Mathematical Modelling, vol. 35, no. 2, pp. 612-620, 2011.

[25] S. Chen and J. Cao, "Projective synchronization of neural networks with mixed time-varying delays and parameter mismatch," Nonlinear Dynamics, vol. 67, no. 2, pp. 1397-1406, 2012.

[26] Z. Yang and D. Xu, "Stability analysis and design of impulsive control systems with time delay," IEEE Transactions on Automatic Control, vol. 52, no. 8, pp. 1448-1454, 2007. 


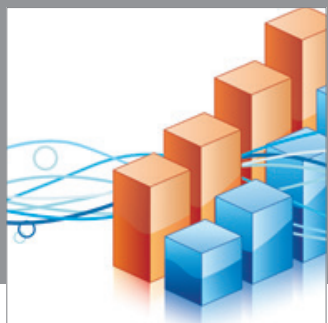

Advances in

Operations Research

mansans

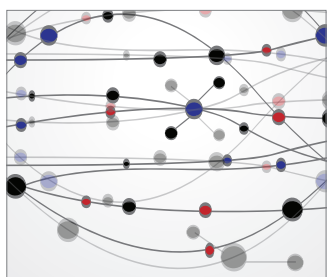

The Scientific World Journal
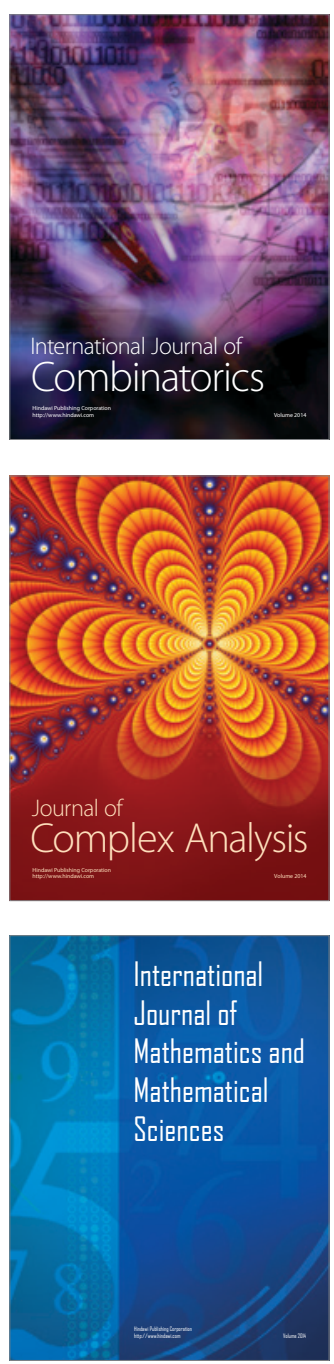
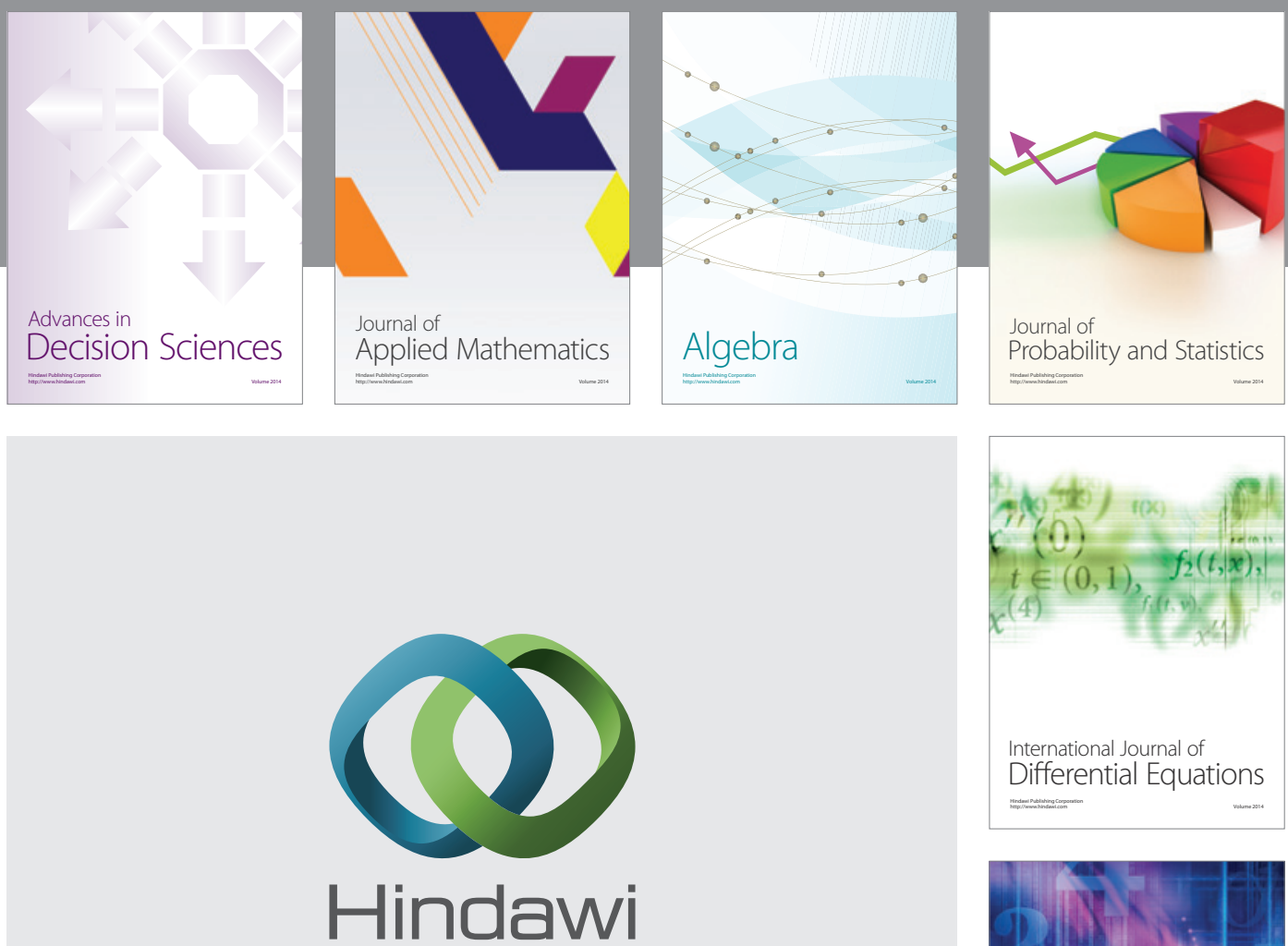

Submit your manuscripts at http://www.hindawi.com
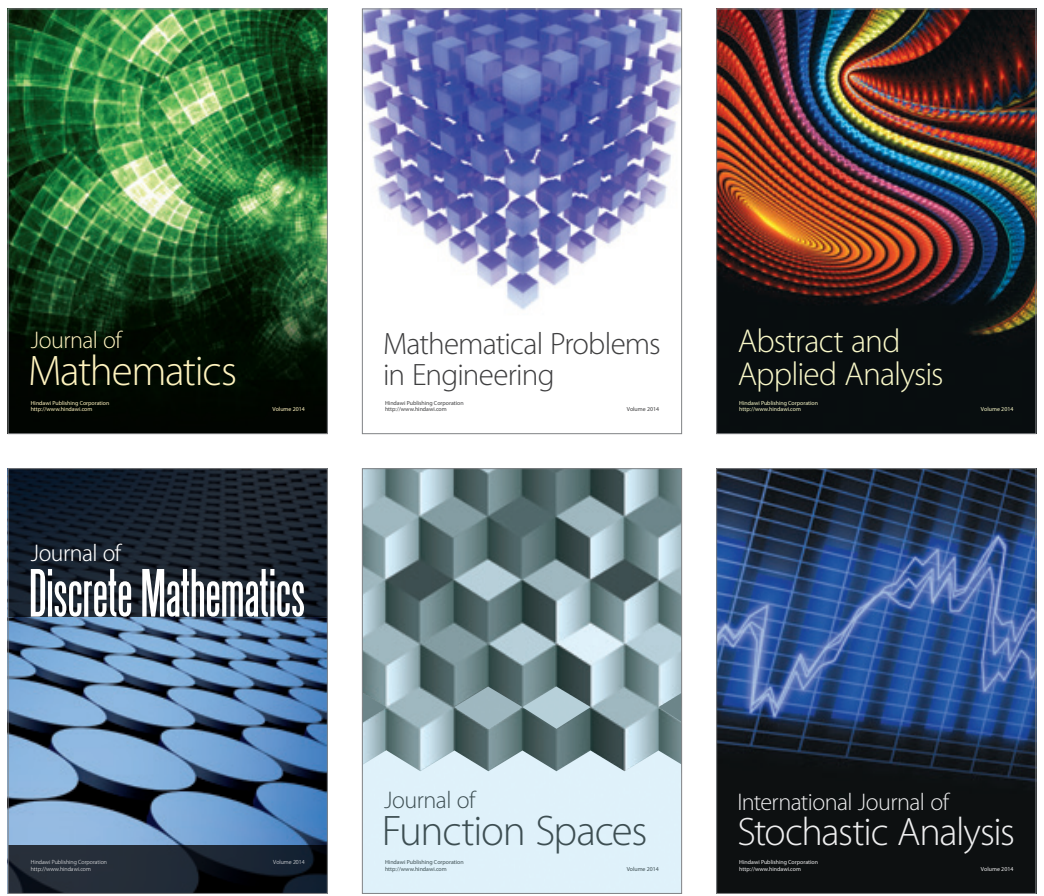

Journal of

Function Spaces

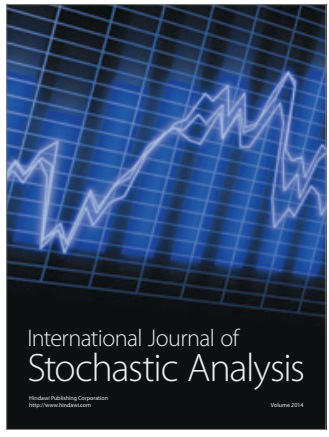

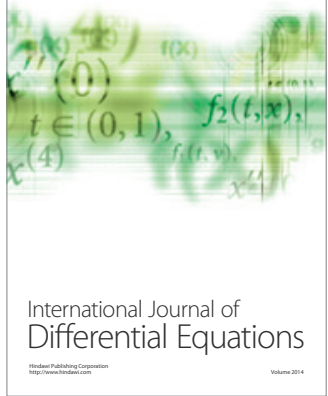
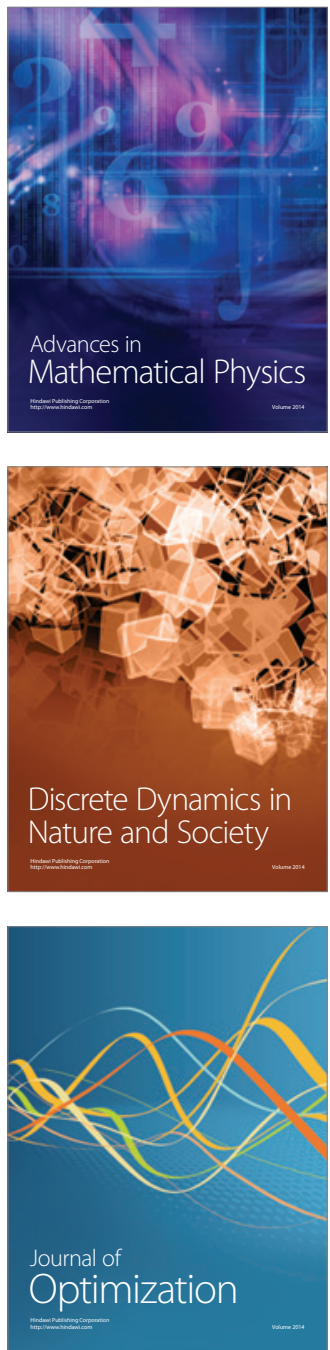